\title{
Microglial activation occurs in the absence of anxiety-like behavior following microembolic stroke in female, but not male, rats
}

\author{
Christina L Nemeth ${ }^{1,2}$, Renuka Reddy ${ }^{2}$, Mandakh Bekhbat ${ }^{2}$, Jabari Bailey ${ }^{2}$ and Gretchen N Neigh ${ }^{1,2^{*}}$
}

\begin{abstract}
Background: The incidence of depression and anxiety disorders is twice as high in women than men; however, females exhibit less neuronal damage following an equivalent ischemic event. Microembolic stroke increases anxiety- and depressive-like behaviors in male rats but the behavioral repercussions in females are unknown.

Findings: Given the relative neuronal protection from stroke in ovary-intact females, female rats exposed to microembolic stroke may be behaviorally protected as compared to males. The data presented demonstrate that anxiety-like behavior is increased in males despite a comparable increase in microglial activation following microembolic stroke in both males and females.

Conclusions: These data suggest that males may be more behaviorally susceptible to the effects of microembolic stroke and further illustrate a dissociation between neuroinflammation and behavior in females.
\end{abstract}

Keywords: Sex differences, Vascular ischemia, Animal model, Behavior, Microglia

\section{Findings}

Though it is unclear whether men and women differ in the presentation of microvascular lesions, women experience reduced incidence of coronary heart disease and stroke [1,2]. Female sex steroids have been shown to garner protection following ischemia putting premenopausal women at an advantage over men with regards to recovery [3]. In cases of traumatic brain injury, women experience reduced secondary edema and better functional outcome compared to men, a finding consistent with rodent models of injury [4]. However, females report a higher incidence of depression and anxiety following stroke [1], and cerebrovascular disease appears to be more depressogenic among women than men [5].

The variable results regarding the influence of sex on outcome from ischemia do not allow for clear generalizations, but indicate that effects observed in males cannot be reliably extrapolated to females. Previous findings show that the induction of microembolic infarcts induces behavioral disruption after long-term recovery in male

\footnotetext{
* Correspondence: gretchen.neigh@emory.edu

'Department of Psychiatry and Behavioral Science, Emory University, Atlanta, GA, USA

2Department of Physiology, Emory University, Atlanta, GA, USA
}

rats [6] and, therefore, the current study builds upon these findings to compare the behavioral and histological effects of microembolic stroke between males and females. Here, we used the same microsphere embolism (ME) model [6] to measure the behavioral and microglial response in both male and female rats to determine whether ME-induced cerebral modifications were consistent between male and female rats. We chose to focus on microglial activation in parallel with anxiety-like behavior because of previous work demonstrating a role of microglial activation in anxiety-like behavior [7-9].

\section{Materials and methods}

Adult male and female Wistar rats (3 months of age, Charles River, Wilmington, MA, USA) were pair-housed by sex until surgery. An AAALAC-approved facility maintained the rats on a reverse 14:10 light:dark cycle in a temperature- and humidity-controlled vivarium with ad libitum food and water. We performed all experiments in accordance with the Institutional Animal Care and Use Committee of Emory University and the National Institutes of Health Guide for the Care and Use of Laboratory Animals. Following at least a 1-week acclimation period, rats were randomly assigned to sham 

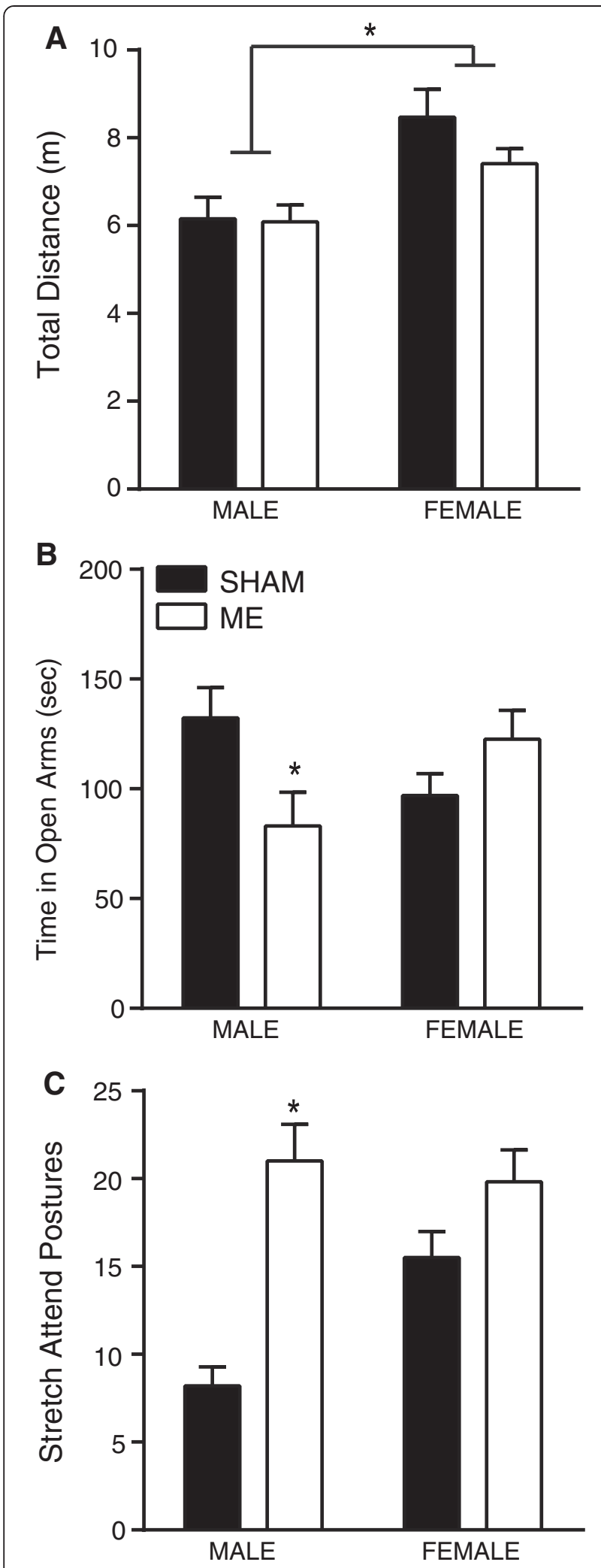

Figure 1 Male and female sham and microsphere embolism (ME) rats were run in a 5-minute elevated plus maze. (A) Total distance traveled of male and female sham and ME rats indicated a significant effect of sex, such that female rats traveled more overall as compared to male rats $(P<0.05)$. (B) A significant interaction between sex and surgery was detected in the elevated plus maze: specifically, compared to male sham, male ME rats spent less time in the open arms of the elevated plus maze, indicative of an anxietylike state. No difference was detected between female sham and ME rats $(P<0.05)$. (C) Similarly, the number of stretch attend postures were significantly higher in male ME rats compared to both sham and female rats $(P<0.05)$. For all, error bars represent standard error of the mean (SEM) and *indicates $P<0.05$.

(male $\mathrm{n}=10$; female $\mathrm{n}=10$ ) or microsphere embolism (ME; male $\mathrm{n}=10$; female $\mathrm{n}=11$ ) surgical groups. For ME surgeries, rats were isoflurane anesthetized and secured in a supine position. A neck incision was made and the common carotid artery was isolated and ligated followed by suture ligation of the external carotid at the bifurcation with the internal carotid artery. Microspheres (New England Nuclear Inc., Boston, MA, USA; $50 \mu \mathrm{m}$ in diameter; suspended in $10 \%$ dextran and $0.01 \%$ Tween in isotonic saline; approximately 2,500 spheres in $50 \mu \mathrm{l}$ ) were injected, using a 30-G needle, into the left internal carotid artery. Female estrous cycle stages were tracked by vaginal lavage prior to surgery and surgeries were performed during diestrus.

The elevated plus maze (EPM) was used as a measure of anxiety-like behavior which consisted of a 5-minute exposure on Day 14 during the animals' dark cycle. While general locomotor behavior did not differ as a result of ME ( $P>0.05$; Figure $1 \mathrm{~A})$, females (sham and ME) were more active in the EPM compared to male rats $\left(\mathrm{F}_{1,36}=14.92, P<0.05\right)$. Furthermore, analysis of time in the open arms of the EPM revealed a significant interaction of sex and surgery, such that ME procedures affected male, but not female, behavior at 2 weeks $\left(\mathrm{F}_{1,43}=\right.$ 7.140, $P<0.05$; Figure 1B). Bonferroni posttests revealed a difference between male sham and male ME time spent in the open arms of the maze (mean difference: $49.03 \mathrm{~s}, P<0.05)$. Females that underwent the ME procedure did not demonstrate any evidence of increased anxiety-like behavior as defined by differences in time spent in the open arms of the maze, or in stretch attend postures. Conversely, males had an increased display of stretch attend postures compared to sham-operated male and female rats (interaction: $\mathrm{F}_{1,42}=7.004, P<0.05$; posttest: mean difference: $12.80, P<0.01$; Figure $1 C$ ).

Twenty-four hours following EPM behavior, rats were transcardially perfused with $4 \%$ paraformaldehyde and brains were removed and stored at $4{ }^{\circ} \mathrm{C}$ until sectioned at $40 \mu \mathrm{M}$. Sections (section sampling frequency $=12$ ) were taken for IBA1 (hippocampus, caudate nucleus, amygdala) immunohistochemistry and cell morphology. IBA1 
(rabbit anti-IBA1, 1:500; Wako, Richmond, VA, USA) sections were stained and analyzed as previously described [6]. IBA1 count data in the hippocampus, amygdala, and caudate nucleus are expressed as the estimated number of cells. Cell morphology in the hippocampus was assessed from the same sections in which 25 to 30 cells were chosen at random, converted to 8-bit, cleaned with a Gaussian filter, binarized, and analyzed for the number of branches, the number of junctions, and average branch length using the ImageJ AnalyzeSkeleton plugin (National Institutes of Health, version 1.47). For all analyses, the investigator was blind to treatment group.

A 2-way ANOVA confirms that in the hippocampus, both male and female rats showed increased estimated counts of IBA1+ cells following ME surgery (main effect of surgery: $F_{1,14}=8.018, P<0.05$; Figure $2 \mathrm{~A}$ ). Conversely, a main effect of sex was present in the amygdala $\left(\mathrm{F}_{1,11}=7.68\right.$, $P<0.05$; Figure 2B) indicating an overall decreased microglial detection in both sham and ME female rats. Though no differences were detected via a 2-way ANOVA in the caudate nucleus, separate examination of male and female rats using a priori student's $t$-test revealed an increase in female IBA1 cells following ME and suggests that the cellular response in the caudate nucleus may be more sensitive in females compared to males $(\mathrm{t}(6)=3.40, P<0.05$; Figure $2 \mathrm{C}$ ).

A 2-way ANOVA of the morphology of IBA1+ cells in the hippocampus showed no effect of sex; however, ME surgery significantly increased both the number of branches $\left(\mathrm{F}_{1,12}=7.73, P<0.05\right.$; Figure $\left.2 \mathrm{D}\right)$ as well as the number of junctions $\left(\mathrm{F}_{1,12}=7.57, P<0.05\right)$ without affecting the average branch length $(P>0.05)$. Representative cells are depicted for sham and ME rats in Figures $2 \mathrm{E}$ and $2 \mathrm{~F}$, respectively.

\section{Conclusions}

Collectively, these data demonstrate that males are more susceptible than females to the behavioral effects of ME and suggest that microglial activation is brain region
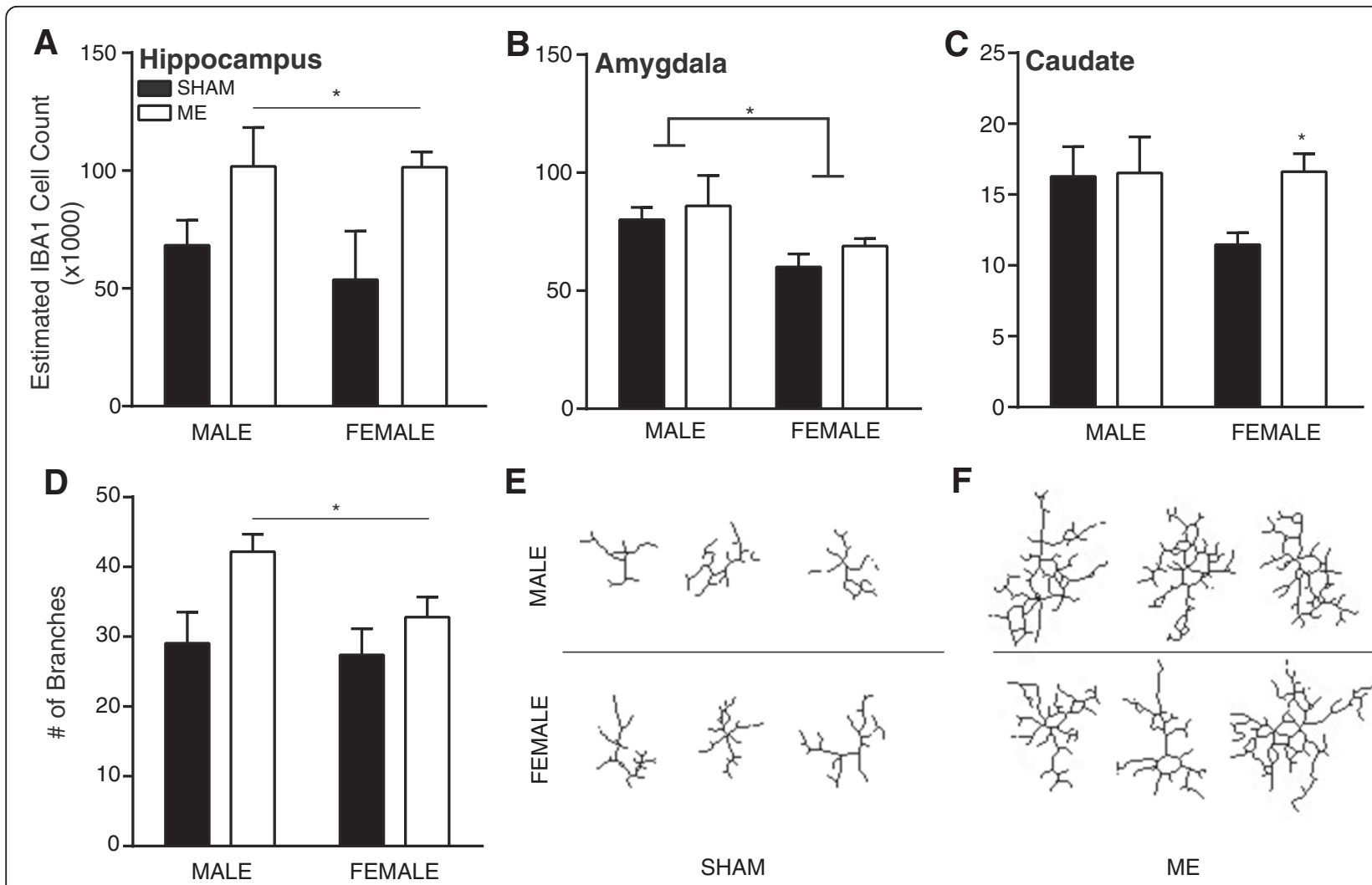

$\mathbf{E}$
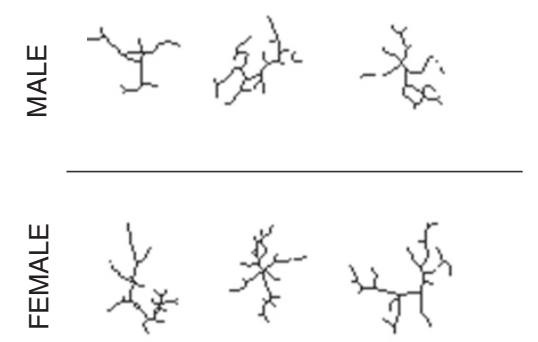

SHAM
$\mathbf{F}$

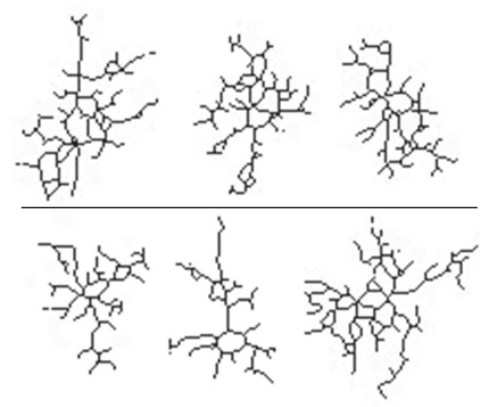

ME

Figure 2 In depth microglia/macrophage activation was determined by cellular counts of ionized calcium-binding adapter molecule 1 (IBA1)-stained cells in the brains of male and female sham and ME rats. (A) IBA1 cell counts were estimated in the hippocampus of male and female sham and ME rats and compared with a 2-way ANOVA. Analysis revealed a main effect of surgery such that ME procedures increased the number of activated microglial cells independent of sex. (B) In contrast, staining in the amygdala revealed an effect of sex, with females having fewer overall IBA $1+$ cells as compared to males $(P<0.05)$. (C) A priori $t$-test of the estimated cell counts in the caudate nucleus showed and increased number of stained cells $(P<0.05)$; through higher at baseline, males showed no effect of ME. (D) Morphological assessment of IBA $1+$ cells in the hippocampus illustrated increased branching and a hyper-ramified state in both male and female ME rats compared to sex-matched sham animals $(P<0.05)$. Representative cells for male and female sham $(\mathbf{E})$ and $\mathrm{ME}$ rats $(\mathbf{F})$ are shown. For all, error bars represent standard error of the mean (SEM) and *indicates $P<0.05$. 
specific and is not coupled to anxiety-like behavior in female rats. In regard to the males, the data extend previous documentation of the behavioral effects of ME to include an additional metric of anxiety-like behavior, the elevated plus maze. Though our previous studies document anhedonia, social and anxiety-like dysfunctions, in addition to the effects of ME on lesion volume and the general presence of microglia [6], our current work refines our understanding of the histological effect of ME by documenting an increase in reactive microglia and an altered morphology of those cells. A causal relationship between microglial activation and anxiety-like behavior has been demonstrated following global ischemia [7] and these data suggest that a similar relationship may exist following ME in male rats.

The absence of altered behavior following ME infarction in females, despite clear evidence of microglial activation, may illustrate a resilience that stems from the neuroprotective effects of endogenous estrogen. Though we detected no behavioral abnormalities in females following ME, it is possible that behavioral deficits exist in other metrics of behavior not measured here, and that these deficits are linked to inflammation within the caudate nucleus and/or hippocampus. Clinical and rodent literature support the role of estrogen as a neuroprotectant following stroke or other brain trauma $[3,10]$ and studies of other rodent ischemia models note reduced infarct size in female rodents compared to both males and ovariectomized female counterparts [11] (thoroughly reviewed in [2]), though no studies have examined the long-term effects of microvascular injury in male and female rats. We have previously reported that frank lesions are rare following ME procedure in male rats [6] and, in the current study, we demonstrate that microglial activation is region dependent, and that microglia within the hippocampus adopt a hyperramified state. The enhanced ramification, illustrated by increased branching and junctions observed in these cells, is likely a heightened response to ME that stems from systemic inflammation and serves to protect the damaged area from additional injury [12-14]. Importantly, these specific cellular adaptations do not associate with behavior suggesting that the mechanism for the behavioral difference within the current examination is not at the level of neuronal damage. The possibility remains that female sex steroids may be modifying the functional consequences of ME-induced microglial activation as has been reported for chronic stress [15]. Interactions among estrogen and microglia are diverse and plentiful [15], and estrogen has been shown to simultaneously stimulate microglia and neural repair [16]. Alternatively, sex differences in neuronal or signaling reorganization and plasticity unrelated to microglia/macrophage activation may mediate these differences in behavior [17].
Collectively, these data demonstrate that following microembolic stroke, both male and female rats exhibit evidence of neuroinflammation with increased numbers of 'primed' microglial cells, but that females do not exhibit corresponding anxiety-like behaviors. A relationship between anxiety-like behavior and neuroinflammation has been previously demonstrated in male rats in models of global ischemia [7] and chronic stress $[8,9]$. The current data set suggests that this relationship cannot be extrapolated to the female brain. Future studies investigating the mechanisms by which females exhibit normal behavior in the presence of microglial activation will provide important insight for treatment strategies.

\section{Abbreviations}

ME: microsphere embolism; EPM: elevated plus maze; IBA1: ionized calciumbinding adapter molecule 1 .

\section{Competing interests}

The authors declare that they have no competing interests.

\section{Authors' contributions}

CLN participated in study design, immunohistochemical procedures, analysis and interpretation of data, and drafting of the manuscript. RR and carried out immunohistochemical procedures and quantification. MB participated in immunohistochemical quantification and image collection. JB maintained animals and performed behavioral testing. GNN is responsible for project conception and design, data interpretation, and was involved in critical revision of the manuscript. All authors read and approved the final manuscript.

\section{Acknowledgements}

We would like to thank Darrell Eacret for his assistance with this project.

\section{Funding}

American Heart Association, National Alliance for Research in Schizophrenia and Affective Disorders.

Received: 11 April 2014 Accepted: 28 September 2014

Published online: 06 November 2014

\section{References}

1. Bushnell C, McCullough LD, Awad IA, Chireau MV, Fedder WN, Furie KL, Howard VJ, Lichtman JH, Lisabeth LD, Pina IL, Reeves MJ, Rexrode KM, Saposnik G, Singh V, Towfighi A, Vaccarino V, Walters MR: Guidelines for the prevention of stroke in women: a statement for healthcare professionals from the American Heart Association/American Stroke Association. Stroke 2014, 45:1545-1588.

2. Manwani B, McCullough LD: Sexual dimorphism in ischemic stroke: lessons from the laboratory. Womens Health 2011, 7:319-339.

3. Wagner AK, Willard LA, Kline AE, Wenger MK, Bolinger BD, Ren D, Zafonte $R D$, Dixon CE: Evaluation of estrous cycle stage and gender on behavioral outcome after experimental traumatic brain injury. Brain Res 2004, 998:113-121

4. Xiong Y, Mahmood A, Lu D, Qu C, Goussev A, Schallert T, Chopp M: Role of gender in outcome after traumatic brain injury and therapeutic effect of erythropoietin in mice. Brain Res 2007, 1185:301-312.

5. Wendell C, Hosey M, Lefkowitz D, Katzel L, Siegel E, Rosenberger W, Waldstein S: Depressive symptoms are associated with subclinical cerebrovascular disease among healthy older women, not men. Am J Geriatr Psychiatry 2010, 18:940-947.

6. Nemeth CL, Shurte MS, McTique DM, Nemeroff CB, Neigh GN: Microembolism infarcts lead to delayed changes in affective-like behaviors followed by spatial memory impairment. Behav Brain Res 2012. 234:259-266.

7. Neigh GN, Karelina K, Glasper ER, Bowers SLK, Zhang N, Popovich PG, DeVries AC: Anxiety after cardiac arrest/cardiopulmonary resuscitation: 
exacerbated by stress and prevented by minocycline. Stroke 2009, 40:3601-3607.

8. Wohleb ES, Powell ND, Godbout JP, Sheridan JF: Stress-induced recruitment of bone marrow-derived monocytes to the brain promotes anxiety-like behavior. J Neurosci 2013, 33:13820-13833.

9. Wohleb ES, Patterson JM, Sharma V, Quan N, Godbout JP, Sheridan JF: Knockdown of interleukin-1 receptor type-1 on endothelial cells attenuated stress-induced neuroinflammation and prevented anxiety-like behavior. J Neurosci 2014, 34:2583-2591.

10. Li J, Siegel M, Yuan M, Zeng Z, Finnucan L, Persky R, Hurn PD, McCullough LD: Estrogen enhances neurogenesis and behavioral recovery after stroke. J Cereb Blood Flow Metab 2011, 31:413-425.

11. Alkayed NJ, Harukuni I, Kimes AS, London ED, Traystman RJ, Hurn PD, Grady $P A:$ Gender-linked brain injury in experimental stroke - editorial comment. Stroke 1998, 29:159-166

12. Hinwood M, Tynan RJ, Charnley JL, Beynon SB, Day TA, Walker FR: Chronic stress induced remodeling of the prefrontal cortex: structural reorganization of microglia and the inhibitory effect of minocycline. Cereb Cortex 2013, 23:1784-1797.

13. Moisse K, Welch I, Hill T, Volkening K, Strong MJ: Transient middle cerebral artery occlusion induces microglial priming in the lumbar spinal cord: a novel model of neuroinflammation. J Neuroinflamm 2008, 5:29.

14. Perry VH, Holmes C: Microglial priming in neurodegenerative disease. Nat Rev Neurol 2014, 10:217-224.

15. Pyter LM, Kelly SD, Harrell CS, Neigh GN: Sex differences in the effects of adolescent stress on adult brain inflammatory markers in rats. Brain Behav Immune 2013, 30:88-94.

16. Zhang L, Nair A, Krady K, Corpe C, Bonneau R, Simpson I, Vannucci S: Estrogen stimulates microglia and brain recovery from hypoxia-ischemia in normoglycemic but not diabetic female mice. J Clin Invest 2004, 113:85-95.

17. Smith AL, Alexander M, Rosenkrantz TS, Sadek ML, Fitch RH: Sex differences in behavioral outcome following neonatal hypoxia ischemia: insights from a clinical meta-analysis and a rodent model of induced hypoxic ischemic brain injury. Exp Neurol 2014, 254:54-67.

doi:10.1186/s12974-014-0174-7

Cite this article as: Nemeth et al:: Microglial activation occurs in the absence of anxiety-like behavior following microembolic stroke in female, but not male, rats. Journal of Neuroinflammation 2014 11:174.

\section{Submit your next manuscript to BioMed Central and take full advantage of:}

- Convenient online submission

- Thorough peer review

- No space constraints or color figure charges

- Immediate publication on acceptance

- Inclusion in PubMed, CAS, Scopus and Google Scholar

- Research which is freely available for redistribution

Submit your manuscript at www.biomedcentral.com/submit
(O) Biomed Central 